\title{
SARS-CoV-2 Receptors are Expressed on Human Platelets and the Effect of Aspirin on Clinical Outcomes in COVID-19 Patients
}

\section{Aditya Sahai}

Cleveland Clinic Lerner College of Medicine

\section{Rohan Bhandari}

Cleveland Clinic

Milka Koupenova

University of Massachusetts Lowell

Jane Freedman

University of Massachusetts Lowell

Mathew Godwin

Cleveland Clinic

Thomas Mclntyre

Cleveland Clinic

Mina Chung

Cleveland Clinic

Jean-Pierre Iskandar

Cleveland Clinic

Hayaan Kamran

Cleveland Clinic Lerner College of Medicine

Anu Aggarwal

Cleveland Clinic

Ankur Kalra

Cleveland Clinic

John Bartholomew

Cleveland Clinic

Keith McCrae

Cleveland Clinic

Ayman Elbadawi

The University of Texas Medical Branch at Galveston

Lars Svensson

Cleveland Clinic

Samir Kapadia 
Cleveland Clinic

\section{Essa Hariri}

Cleveland Clinic

Scott Cameron ( $\sim$ cameros3@ccf.org )

Cleveland Clinic

\section{Research Article}

Keywords: Platelets, SARS-CoV-2, COVID-19, Thrombosis, ACE2, TMPRSS2

Posted Date: December 23rd, 2020

DOI: https://doi.org/10.21203/rs.3.rs-119031/v1

License: (c) (1) This work is licensed under a Creative Commons Attribution 4.0 International License. Read Full License 


\section{SARS-CoV-2 Receptors are Expressed on Human Platelets and the Effect of Aspirin on Clinical}

\section{Outcomes in COVID-19 Patients}

Aditya Sahai $\mathrm{MD}^{1 \ddagger}$, Rohan Bhandari $\mathrm{MD}^{1,2 \ddagger}$, Milka Koupenova $\mathrm{PhD}^{3}$, Jane E. Freedman $\mathrm{MD}^{3}$, Matthew Godwin BS ${ }^{1}$, Thomas Mclntyre PhD ${ }^{2,4}$, Mina K. Chung MD ${ }^{1,2,4}$, Jean-Pierre Iskandar $\mathrm{MD}^{5}$, Hayaan Kamran $\mathrm{MD}^{1}$, Essa Hariri $\mathrm{MD}^{5}$, Anu Aggarwal PhD², Ankur Kalra $\mathrm{MD}^{1}$, John R. Bartholomew $\mathrm{MD}^{1,4}$, Keith R. McCrae MD ${ }^{4,6}$, Ayman Elbadawi MD, Lars G. Svensson MD PhD ${ }^{1,4}$, Samir Kapadia $\mathrm{MD}^{1,4}$ and Scott J. Cameron MD PhD $\mathrm{P}^{1,2,4}$ *

Running Title: Platelets Express SARS-CoV-2 Receptors \& Aspirin Does Not Protect in COVID-19

\section{Author Affiliations:}

Heart Vascular and Thoracic Institute, Cleveland Clinic, Cleveland, $\mathrm{OH}$

Department of Cardiovascular and Metabolic Sciences, Cleveland Clinic, Cleveland, $\mathrm{OH}$

3 Department of Medicine, University of Massachusetts Medical School, Worcester, MA.

4 Case Western Reserve University Cleveland Clinic Lerner College of Medicine, Cleveland, $\mathrm{OH}$

5 Department of Medicine, Cleveland Clinic, Cleveland, $\mathrm{OH}$

6 Taussig Cancer Institute, Cleveland Clinic, Cleveland, $\mathrm{OH}$

7 Division of Cardiovascular Medicine, University of Texas Medical Branch, Galveston, TX

${ }^{\ddagger}$ Equally contributing authors

Word Count: 2995 (main text), 199 (abstract)

\section{References: 55}

Figures: 4

\section{Tables: 2}

Address for Correspondence*: Cleveland Clinic Foundation, Department of Cardiovascular Medicine, Section of Vascular Medicine, J3-5, 9500 Euclid Avenue, Cleveland, OH, 44195, USA. Telephone: (216) 316-6794. Email: cameros3@ccf.org

Key Words: Platelets, SARS-CoV-2, COVID-19, Thrombosis, ACE2, TMPRSS2 


\section{Abstract}

34 Coronavirus disease-2019 (COVID-19) caused by SARS-CoV-2 is an ongoing viral pandemic marked by

35 increased risk of thrombotic events. However, the role of platelets in the elevated observed thrombotic

36 risk in COVID-19 and utility of anti-platelet agents in attenuating thrombosis is unknown. We aimed to

37 determine if human platelets express the known SARS-CoV-2 receptor-protease axis on their cell surface

38 and assess whether the anti-platelet effect of aspirin may mitigate risk of myocardial infarction (MI),

39 cerebrovascular accident (CVA), and venous thromboembolism (VTE) in COVID-19. Expression of ACE2

40 and TMPRSS2 on human platelets were detected by immunoblotting and confirmed by confocal

41 microscopy. We evaluated 22,072 symptomatic patients tested for COVID-19. Propensity-matched

42 analyses were performed to determine if treatment with aspirin or non-steroidal anti-inflammatory

43 drugs (NSAIDs) affected thrombotic outcomes in COVID-19. Neither aspirin nor NSAIDs affected

44 mortality in COVID-19. However, both aspirin and NSAID therapies were associated with increased risk

45 of the combined thrombotic endpoint of (MI), (CVA), and (VTE). Thus, while platelets clearly express

46 ACE2-TMPRSS2 receptor-protease axis for SARS-CoV-2 infection, aspirin does not prevent thrombosis

47 and death in COVID-19. The mechanisms of thrombosis in COVID-19, therefore, appears distinct and the

48 role of platelets as direct mediators of SARS-CoV-2-mediated thrombosis warrants further investigation. 
Introduction

58 COVID-19 is caused by the severe acute respiratory syndrome coronavirus-2 (SARS-CoV-2) and curiously

59 displays a propensity for thrombosis in multiple vascular beds. COVID-19-related thrombosis may

60 contribute to severe organ injury and death. The incidence of thrombotic events was as high as $31 \%$ in

61 one cohort ${ }^{1}$. Clinical and autopsy studies of COVID-19 patients suggest an increased risk of

62 microthrombi, venous thromboembolism (VTE), and ischemic stroke ${ }^{2,3}$. Activated platelets are

63 circulating mediators of thrombosis and, therefore, may serve as a logical therapeutic target in COVID-

64 19. Two registered clinical trials (NCT04363840 and NCT04365309) will prospectively evaluate patient

65 outcomes following low dose aspirin in the context of SARS-CoV-2 infection.

67 SARS-CoV-2 utilizes an spike glycoprotein to bind to the host transmembrane angiotensin-converting enzyme 2 (ACE2) and is then cleaved by the serine protease TMPRSS2 to coordinate entry into the host

69 cell ${ }^{4,5}$. Therefore, co-expression of ACE2 and TMPRSS2 may be important for host cell entry and

70 infectivity of SARS-CoV-2. Importantly, human tissue distribution of ACE2 and TMPRSS2 mirrors organ

71 system involvement in COVID-19 and includes the lungs $\mathrm{s}^{6-11}$, vascular endothelium ${ }^{9-12}$, heart $^{11,13,14}$,

72 kidneys $^{8,10,13}$, liver ${ }^{8,10}$, digestive tract ${ }^{8,10,11,15}$, nasal epithelium ${ }^{7,10,11}$ and central nervous system ${ }^{10,14}$.

73 Single-stranded RNA (ssRNA) viruses, including influenza, are engulfed by platelets and may contribute

74 to immuno-thrombosis indirectly through developing neutrophil extracellular traps (NETs) by engaging

75 the platelet toll-like receptor $7(\mathrm{TLR} 7)^{16}$. SARS-CoV-2, another SSRNA virus, utilizes platelets to modulate

76 immunologic responses including the development of neutrophil extracellular traps (NETs) that are

77 emerging as pro-thrombotic responses in patients with COVID-19 ${ }^{17}$. Further, elevation of soluble P-

78 selectin and SCD40L in blood from patients with COVID-19 compared to controls provides indirect

79 evidence of platelet activation in COVID-19 coagulopathy ${ }^{18}$. SARS-CoV-2 is a ssRNA virus, and therefore

80 may directly augment platelet activation causing myocardial infarction (MI), stroke, and VTE. 
82 A recent report demonstrated that COVID-19 patients have a divergent platelet transcriptome from

83 healthy individuals, and aspirin suppresses COVID-19 platelet activation in vitro ${ }^{19}$. The platelet surface

84 receptor for SARS-CoV-2 was not clarified in this study, while a similar investigation by another group

85 identified mRNA for SARS-CoV-2 in human platelets ${ }^{20}$. Thus, our goal was to determine if platelets

86 express known SARS-CoV-2 receptor proteins and, as with influenza previously, contribute to thrombotic

87 events in patients. In the absence of clinical trial data, we sought to evaluate the potential benefit in

88 mitigating thrombotic responses in vivo with use of aspirin or other NSAID antiplatelet therapies by

89 propensity matching patients using real-world data.

90

\section{$91 \quad$ Methods}

\section{Platelet Isolation}

93 Healthy volunteers without any known medical history or on antiplatelet therapy donated blood

94 specimens in accordance with and approved by the Cleveland Clinic Foundation Institutional Review

95 Board (IRB) approval. For each subject, venous blood was drawn by a medical professional into citrate

96 plasma tubes, then centrifuged in a tabletop centrifuge at 1100 RPM for 15 minutes. The platelet rich

97 plasma (PRP), collected well above the buffy coat, was decanted and the platelets were centrifuged at

982600 RPM for an additional 5 minutes. These washed platelets were then used in immunoblotting and

99 fluorescence-activated cell sorting (FACS) analyses.

100

101 Immunoblotting

102 Washed platelets from healthy subjects or patients with coronary artery disease (CAD) enrolled at the

103 Cleveland Clinic main campus in Ohio were isolated and proteins separated by SDS-PAGE as we have

104 previously documented ${ }^{21,22}$ and in accordance with IRB protocols (\#19-1451 for patients and \#20-413 
105 for healthy volunteers). We utilized human brain lysate, human placenta, and engineered human heart

106 tissue as positive controls for TMPRSS2 and ACE2. Human brain lysate is commercially available (Novus

107 \#NB820-59177). Human placenta lysate was prepared as follows: placental villous tissue was collected

108 immediately upon uncomplicated, full-term (37-42 weeks' gestation), elective C-section deliveries at

109 MetroHealth Hospital in Cleveland, Ohio and approved by the Cleveland Clinic and MetroHealth IRB

110 (\#16-1311 and \#16-00335, respectively). This tissue was normally discarded placentas with intact fetal

111 membranes, and following inclusion in the study no protected health information, identifiers, or clinical

112 data were collected. A waiver of consent was approved by the Cleveland Clinic Foundation IRB as

113 the placentas were collected anonymously. Engineered human heart tissue was obtained as follows:

114 human-induced pluripotent stem cells (generated by the California Institute of Regenerative Medicine)

115 were differentiated into beating ventricular-like cardiomyocytes (iCMs) and grown in a monolayer. To

116 enhance maturation, iCMs were subsequently grown as engineered heart tissues as we have previously

117 described ${ }^{23}$. Immunoblotting was conducted using anti-TMPRSS2 (abcam \#92323), anti-ACE2 (Abcam

118 \#15348), anti-tubulin (CST \#3873S), and anti-GAPDH (CST \#5174) antibodies. The mean ratio of

119 TMPRSS2 or ACE2 to loading control \pm SEM is documented, unless stated otherwise. Primary antibody

120 was used as in a 1:10000 titer overnight at $4{ }^{\circ} \mathrm{C}$ in $3 \%$ bovine serum albumin/Tris-buffered saline-Tween

121 20. Secondary antibody (GE Healthcare, Buckinghamshire, UK) was used in a 1:2000 titer in 5\%

122 milk/Tris-buffered saline-Tween for 1 hour at room temperature. Final autoradiographic films (Bioblot

123 BXR, Laboratory Product Sales, Rochester, NY) were quantified by densitometry using ImageJ software

124 (National Institutes of Health). All experiments were performed in accordance with relevant guidelines

125 and regulations.

126

127

128 


\section{Confocal Microscopy}

130 Venous blood drawn into and separated as citrated plasma was lysed and fixed with BD FACS lysing 131 solution (BD Biosciences, NJ, USA, cat\# 349202) for 10 mins. The platelet pellet was washed with $1 X$ 132 PBS, centrifuged at 1500g for 7 mins, resuspended in HEPES-buffered Tyrode solution supplemented 133 with 2\% FBS and then stained for 1 hour with the following: CD41 to confirm platelets (ThermoFisher 134 eBio cat \#11-0419-42), ACE2 antibody (Novus cat\#NBP2-72117AF647), TMPRSS2 antibody (SantaCruz 135 cat\#sc-515727 AF488) and DAPI to eliminate any DNA components. Mounted slides were resolved by 136 fluorescent microscopy using a Scanning Disk Nikon A1 confocal microscope with 100x objective lens.

137 All experiments were performed in accordance with relevant guidelines and regulations.

\section{Study Design}

140 Quality-assured clinical data from ambulatory and hospitalized Cleveland Clinic patients treated in 141 Northeast Ohio and South Florida was used to appraise data on 22,072 symptomatic patients evaluated 142 for COVID-19 with the goal of determining whether current aspirin use protects patients from death 143 and/or the secondary composite outcome of $\mathrm{Ml}$, thrombotic stroke, and/or VTE. Positive testing for a 144 SARS-CoV-2 amplicon by nasopharyngeal RT-PCR was used to determine infection status. The electronic 145 medical record and hospital Medication Administration Record (MAR) was used to confirm new or 146 ongoing administration of $81 \mathrm{mg}$ aspirin or other NSAIDs for both outpatients and inpatients.

148 Statistical Analysis

149 Categorical factors are summarized using frequencies and percentages, while continuous factors are 150 described using median and ranges. Initial descriptive analyses were performed. Comparisons were 151 made between those with known death status and those with missing death information to identify if 152 any differences exist in these cohorts. Then among those with known death status, differences in COVID 
153 positive and COVID negative patients were assessed. Finally, after stratifying by COVID status,

154 comparisons of those with and without aspirin use were performed. For all tables, continuous measures

155 were compared using nonparametric Wilcoxon rank sum tests, while categorical factors were compared

156 using Pearson chi-square tests or Fisher exact tests, for rare events.

158 Given the differences across many covariates, propensity score matching was performed to account for

159 differences between those with and without aspirin use. This approach used two steps. First, multiple

160 imputation was performed on all demographic and covariate measures within COVID status stratified

161 datasets, using fully conditional specification methods. Ten imputed datasets were created. Then

162 propensity score models were fit for each dataset, with aspirin use as the response and all other

163 measures as predictors. Predicted probability of aspirin use from each model was calculated, and these

164 probabilities were averaged across models for each patient. Greedy matching was then performed

165 using a caliper of 0.2 standard deviations of the logit to create matched datasets for both COVID positive

166 and negative patients. A small number of aspirin users could not be matched well and were excluded

167 from the matched analysis. Comparisons of outcomes were performed using mixed effect logistic

168 regression models to account for the matching process. Overlap weighting propensity score analyses

169 were also performed ${ }^{24}$ which data with the same conclusions. This analysis was repeated using NSAID

170 groups. For significant effects, E-values ${ }^{25}$ that represent the magnitude of the association between an

171 unobserved covariate and both the medication group and outcome necessary to make the result non-

172 significant was also calculated. Analyses were performed using SAS software (version 9.4; Cary, NC). A

173 significance level of 0.05 was assumed for all tests.

174

175

176 


\section{Results}

178 Expression of ACE2 $(n=6)$ and TMPRSS2 $(n=3)$ on the platelet surface was observed by confocal

179 microscopy (Figure 1). Expression of TMPRSS2 in healthy subjects (mean age $40.1 \pm 2.8$ years, $n=20$ ) was

180 also confirmed by immunoblotting at the expected molecular weight of $\sim 50 \mathrm{KDa}$.

181
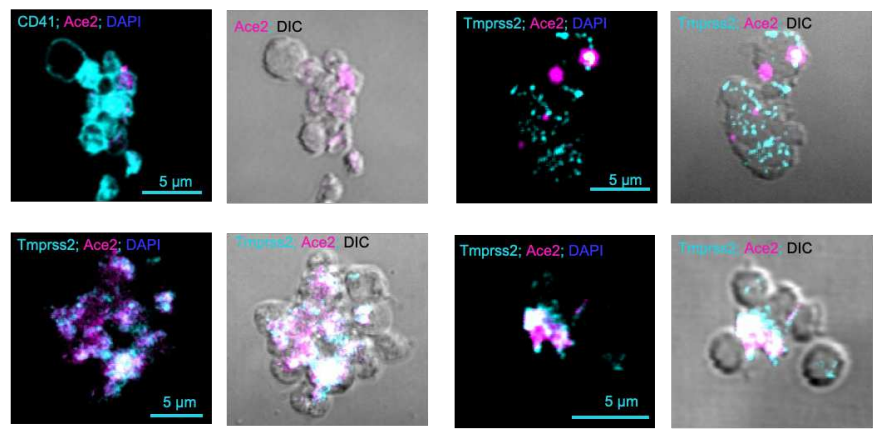

Figure 1. Expression of ACE2 and TMPRSS2 in Platelets by Confocal Microscopy. Platelets isolated from venous blood of healthy individuals was stained for $1 \mathrm{~h}$ with the following antibodies: CD41 (platelet-specific marker), ACE2, TMPRSS2, and DAPI to eliminate any DNA components. Mounted slides were resolved by confocal fluorescent microscopy using a $100 x$ objective lens. Images are representative of $n=6$ donors for ACE2 and $n=3$ for TMPRSS2. Each image represents a different donor. The scale bar is noted.

184 Utilizing human brain as a positive control, TMPRSS2 expression was standardized to a loading control

185 with no correlation between age and platelet TMPRSS2 expression (Figure $2 A ; r^{2}=0.058, p=0.30$ ). Since ACE2 exists as multiple glycosylated proteins of variable molecular weight ${ }^{26-28}$, human brain ${ }^{29}$, human

187 placenta ${ }^{30}$, and engineered heart tissue ${ }^{31}$ were utilized as positive controls to confirm predominant

188 migration at $\sim 100 \mathrm{kDa}$ as expected. Given that patients with confirmed CAD receive antiplatelet

189 medications according to established guidelines, TMPRSS2 expression for healthy controls ( $n=20$ ) was

190 compared to patients with coronary artery disease (CAD, $n=10)$ and, while numerically greater in CAD,

191 was without a statistically significant difference (Figure $2 A, p=0.15$ ). 
193 Similarly, expression of ACE2 in healthy subjects $(n=20)$ was confirmed by immunoblotting. ACE2

194 expression standardized to tubulin did not correlate with age (Figure $2 \mathrm{~B} ; \mathrm{r}^{2}=0.0039, \mathrm{p}=0.79$ ).
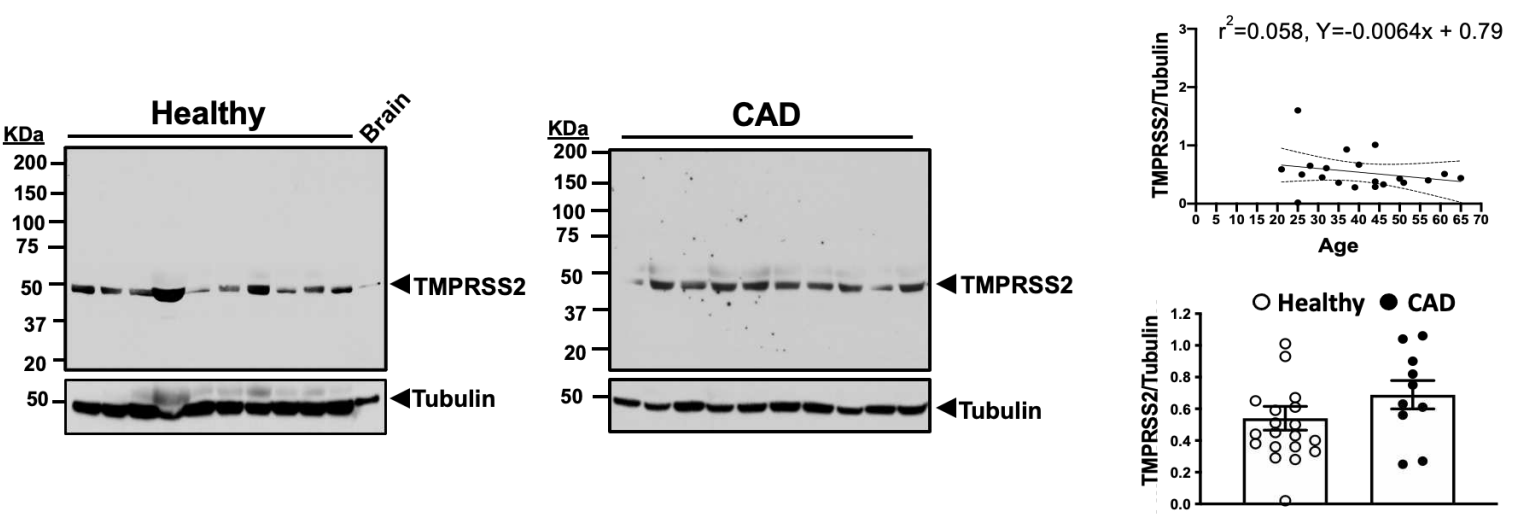

Figure 2A. Expression of TMPRSS2 in Platelets: Washed platelets from healthy individuals (mean age $40.1 \pm$ 2.8 years, $n=20$ ) were isolated and proteins separate by SDS-PAGE with molecular weight shown in KiloDaltons (KDa). Immunoblotting was conducted an using an anti-TMPRSS2 antibody or anti-tubulin immunoblotting as a loading control. The ratio of protein to loading control is expressed as a function of age and the correlation coefficient is noted $(r \pm 95 \% \mathrm{Cl}, \mathrm{P}=0.30)$. Human brain lysate served as a positive control for TMPRSS2 migrating at the expected molecular weight $(\sim 50 \mathrm{KDa})$. Data shown are representative of 20 healthy individuals (10 male and 10 female) and 10 patients with coronary artery disease (CAD). The mean ratio of TMPRSS2/Tubulin \pm SEM is noted, $P=0.145$ between healthy and CAD by Mann Whitney $U$ ). For clarity of presentation, the tubulin blot was cropped just above and below the $50 \mathrm{KDa}$ marker line.

196 Platelet ACE2 in healthy subjects $(n=20)$ was compared to patients with CAD $(n=10)$ and, again, while

197 numerically higher in $C A D$, was without a statistical difference (Figure $2 B, p=0.11$ ). Further, we did not

198 observe sex-specific differences in platelet expression of ACE2 or TMPRSS2 (20 men and 20 women in
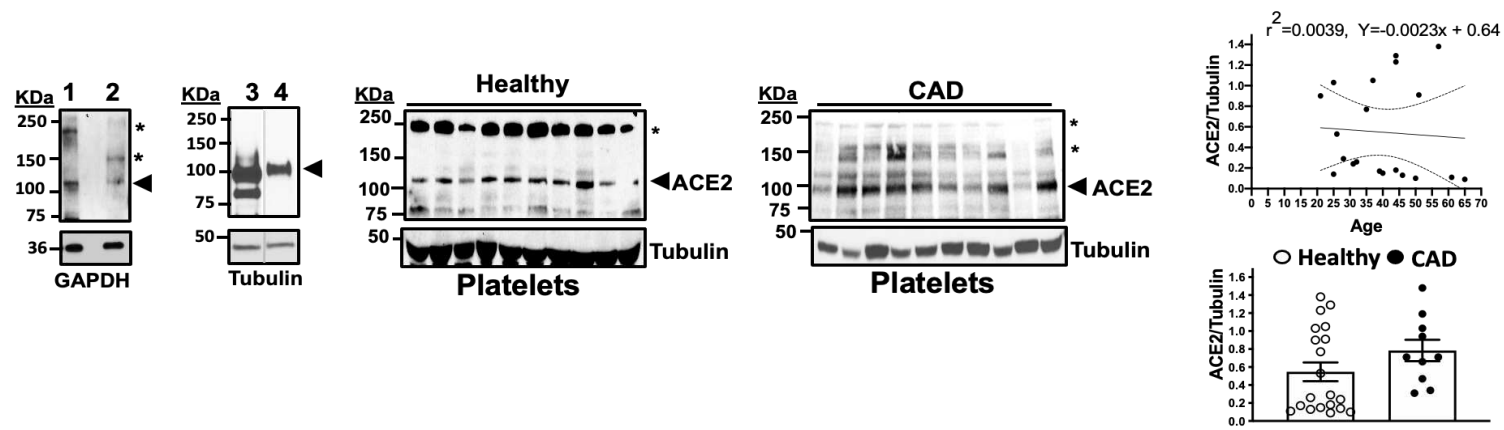

Figure 2B. Expression of ACE2 in Platelets: Washed platelets from healthy individuals (mean age $40.1 \pm 2.8$ years, $n=20$ ) were isolated and proteins separate by SDS-PAGE with molecular weight shown in KiloDaltons $(\mathrm{KDa})$. Lane 1 is human platelet lysate, lane 2 is human brain lysate, lane 3 is human placenta lysate, lane 4 is lysate from engineered human heart tissue. Immunoblotting was conducted using an using anti-ACE2 antibody. Anti-tubulin and anti-GAPDH are loading controls. ACE2 migrates at the expected molecular weight ( 100 KDa) shown by an arrowhead with glycosylated forms indicated by *. The ratio of ACE2 protein to loading control is expressed as a function of age and the correlation coefficient is noted ( $\mathrm{r} \pm 95 \% \mathrm{Cl}, \mathrm{P}=0.79)$. Data shown are representative of 20 healthy individuals (10 male and 10 female) and 10 patients with coronary artery disease (CAD). The mean ratio of ACE2/Tubulin \pm SEM is noted, $P=0.112$ between healthy and CAD by Mann Whitney $U$ ). For clarity of presentation, the tubulin and GAPDH blots are cropped just above and below the $50 \mathrm{Kda}$ and $36 \mathrm{KDa}$ marker lines, respectively and the ACE2 blot is cropped just below the $75 \mathrm{KDa}$ marker. The grey partition line for ACE2 and tubulin are from the same blot separated by three lanes. 
each group). Full size, uncropped immunoblots for ACE2, TMPRSS2, and loading controls are found in

200 Supplemental Figure $1 \mathrm{~A}-\mathrm{C}$.

201

22,072 patients tested for COVID-19 at two Cleveland Clinic hospitals between March 13, 2020 to May

13, 2020 were evaluated. Within this cohort, 11,507 patients had complete clinical data and 1,994

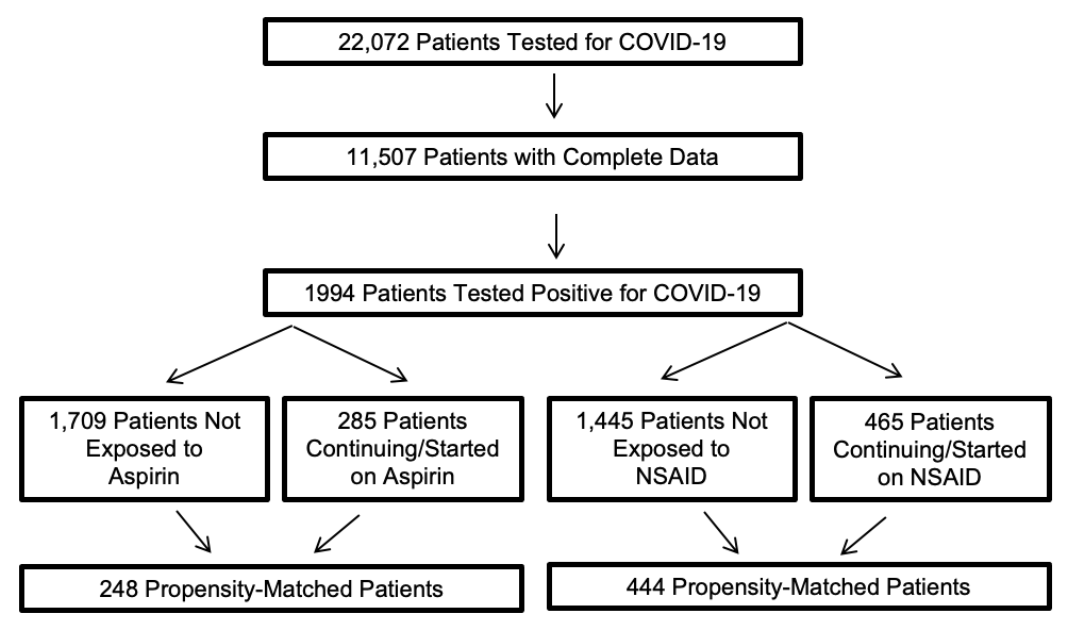

Figure 3. Patients Testing Positive for SARS-CoV-2 taking Aspirin or NSAIDs. Patients testing positive for a SARS-CoV-2 amplicon at two Cleveland Clinic hospitals were evaluated. Patients initiated with aspirin or NSAID therapy or continuing aspirin or NSAID if admitted to the hospital were included in this study. Clinical variables in each group where then re-evaluated following careful propensity matching 
213 Table 1 shows the unadjusted characteristics of each comparative cohort for aspirin.

\begin{tabular}{|c|c|c|c|c|c|}
\hline \multirow[b]{2}{*}{ Factor } & \multicolumn{2}{|c|}{$\begin{array}{l}\text { No Aspirin } \\
(\mathrm{N}=1,709)\end{array}$} & \multicolumn{2}{|c|}{$\begin{array}{c}\text { Aspirin Use } \\
(\mathrm{N}=285)\end{array}$} & \multirow[b]{2}{*}{ p-value } \\
\hline & $\mathbf{N}$ & Statistics & $\mathbf{N}$ & Statistics & \\
\hline \multicolumn{6}{|l|}{ Medications } \\
\hline CLOPID & 1,709 & $9(0.53)$ & 285 & $27(9.5)$ & $<0.001^{c}$ \\
\hline Ticag & 1,709 & $1(0.06)$ & 285 & $6(2.1)$ & $<0.001^{d}$ \\
\hline Prasug & 1,709 & $0(0.00)$ & 285 & $0(0.00)$ & \\
\hline Cangr & 1,709 & $0(0.00)$ & 285 & $0(0.00)$ & \\
\hline Cilost & 1,709 & $0(0.00)$ & 285 & $0(0.00)$ & \\
\hline Pentox & 1,709 & $0(0.00)$ & 285 & $1(0.35)$ & $0.14^{d}$ \\
\hline AntiPlt & 1,709 & $10(0.59)$ & 285 & $285(100.0)$ & $<0.001^{\epsilon}$ \\
\hline Multiple Therapy & 1,709 & $0(0.00)$ & 285 & $34(11.9)$ & $<0.001^{d}$ \\
\hline AC_therputic & 1,709 & $94(5.5)$ & 285 & $56(19.6)$ & $<0.001^{e}$ \\
\hline AC_prophylct & 1,709 & $355(20.8)$ & 285 & $215(75.4)$ & $<0.001^{\epsilon}$ \\
\hline NSAIDs & 1,650 & $294(17.8)$ & 260 & $171(65.8)$ & $<0.001^{c}$ \\
\hline \multicolumn{6}{|l|}{ Covariates } \\
\hline Age & 1,709 & $50.6 \pm 17.5$ & 285 & $70.0 \pm 13.6$ & $<0.001^{a 2}$ \\
\hline Platelets & 689 & $217.4 \pm 79.3$ & 253 & $208.7 \pm 85.3$ & $0.14^{21}$ \\
\hline Gender & 1,651 & & 285 & & $<0.001^{c}$ \\
\hline Male & & $804(48.7)$ & & $172(60.4)$ & \\
\hline Female & & $847(51,3)$ & & $113(39.6)$ & \\
\hline Race & 1,564 & & 280 & & $<0.001^{e}$ \\
\hline White & & $948(60.6)$ & & $144(51.4)$ & \\
\hline Black & & $506(32.4)$ & & $124(44.3)$ & \\
\hline Other & & $110(7.0)$ & & $12(4.3)$ & \\
\hline Ethnicity & 1,480 & & 277 & & $<0.001^{c}$ \\
\hline Hispanic & & $204(13.8)$ & & $7(2.5)$ & \\
\hline Non-Hispanic & & $1,276(86.2)$ & & $270(97.5)$ & \\
\hline Smoking & 1,417 & & 268 & & $<0.001^{e}$ \\
\hline No & & $924(65.2)$ & & $123(45.9)$ & \\
\hline Former & & $362(25.5)$ & & $124(46.3)$ & \\
\hline Current & & $131(9.2)$ & & $21(7.8)$ & \\
\hline RespSuprt & 1,709 & $191(11.2)$ & 285 & $117(41.1)$ & $<0.001^{\epsilon}$ \\
\hline OnPressors & 1,709 & $81(4.7)$ & 285 & $47(16.5)$ & $<0.001^{\epsilon}$ \\
\hline HemodInstab & 1,709 & $85(5.0)$ & 285 & $48(16.8)$ & $<0.001^{c}$ \\
\hline COPD_emphysema & 1,399 & $82(5.9)$ & 274 & $53(19.3)$ & $<0.001^{\epsilon}$ \\
\hline Asthma & 1,410 & $243(17.2)$ & 273 & $66(24.2)$ & $0.007^{\mathrm{c}}$ \\
\hline Diabetes & 1,424 & $318(22.3)$ & 278 & $147(52.9)$ & $<0.001^{e}$ \\
\hline Hypertension & 1,447 & $659(45.5)$ & 281 & $244(86.8)$ & $<0.001^{\epsilon}$ \\
\hline Coronary_artery_disease & 1,405 & $116(8.3)$ & 275 & $100(36.4)$ & $<0.001^{\circ}$ \\
\hline Heart_Failure & 1,404 & $108(7.7)$ & 274 & $78(28.5)$ & $<0.001^{\epsilon}$ \\
\hline Cancer & 1,447 & $184(12.7)$ & 280 & $63(22.5)$ & $<0.001^{e}$ \\
\hline On_immunosuppressive_treatment & 1,456 & $144(9.9)$ & 277 & $36(13.0)$ & 0.12 \\
\hline
\end{tabular}

Statistics presented as Mean \pm SD, N (column \%). p-values: a1=t test, a2 $=$ Satterthwaite t-test, $\mathrm{c}=$ Pearson's chi

square test, $\mathrm{d}=$ Fisher's Exact test. 
215 Table 2 shows the unadjusted characteristics of each comparative cohort for NSAIDs.

\begin{tabular}{|c|c|c|c|c|c|}
\hline \multirow[b]{2}{*}{ Factor } & \multicolumn{2}{|c|}{$\begin{array}{l}\text { No NSAIDs } \\
(\mathrm{N}=1,445)\end{array}$} & \multicolumn{2}{|c|}{$\begin{array}{l}\text { NSAIDs } \\
(\mathrm{N}=465)\end{array}$} & \multirow[b]{2}{*}{ p-value } \\
\hline & $\mathbf{N}$ & Statistics & $\mathbf{N}$ & Statistics & \\
\hline \multicolumn{6}{|l|}{ Medications } \\
\hline CLOPID & 1,445 & $12(0.83)$ & 465 & $21(4.5)$ & $<0.001^{c}$ \\
\hline Ticag & 1,445 & $0(0.00)$ & 465 & $7(1.5)$ & $<0.001^{d}$ \\
\hline Prasug & 1,445 & $0(0.00)$ & 465 & $0(0.00)$ & \\
\hline Cangr & 1,445 & $0(0.00)$ & 465 & $0(0.00)$ & \\
\hline Cilost & 1,445 & $0(0.00)$ & 465 & $0(0.00)$ & \\
\hline Pentox & 1,445 & $0(0.00)$ & 465 & $1(0.22)$ & $0.24^{d}$ \\
\hline AntiPlt & 1,445 & $96(6.6)$ & 465 & $174(37.4)$ & $<0.001^{c}$ \\
\hline Multiple Therapy & 1,445 & $5(0.35)$ & 465 & $26(5.6)$ & $<0.001^{c}$ \\
\hline AC_therputic & 1,445 & $95(6.6)$ & 465 & $45(9.7)$ & $0.026^{x}$ \\
\hline AC_prophylct & 1,445 & $328(22.7)$ & 465 & $203(43.7)$ & $<0.001^{\epsilon}$ \\
\hline \multicolumn{6}{|l|}{ Covariates } \\
\hline Age & 1,445 & $51.5 \pm 18.2$ & 465 & $58.9 \pm 17.1$ & $<0.001^{a}$ \\
\hline Platelets & 574 & $213.1 \pm 80.8$ & 314 & $212.8 \pm 78.0$ & $0.97^{a}$ \\
\hline Gender & 1,390 & & 462 & & $0.36^{\mathrm{c}}$ \\
\hline Male & & $688(49.5)$ & & $240(51.9)$ & \\
\hline Female & & $702(50.5)$ & & $222(48.1)$ & \\
\hline Race & 1,310 & & 451 & & $0.005^{c}$ \\
\hline White & & $801(61.1)$ & & 243 (53.9) & \\
\hline Black & & $416(31.8)$ & & $181(40.1)$ & \\
\hline Other & & $93(7.1)$ & & $27(6.0)$ & \\
\hline Ethnicity & 1,228 & & 454 & & $<0.001^{c}$ \\
\hline Hispanic & & $178(14.5)$ & & $31(6.8)$ & \\
\hline Non-Hispanic & & $1,050(85.5)$ & & $423(93.2)$ & \\
\hline Smoking & 1,162 & & 452 & & $<0.001^{c}$ \\
\hline No & & $763(65.7)$ & & $247(54.6)$ & \\
\hline Former & & $303(26.1)$ & & $160(35.4)$ & \\
\hline Current & & $96(8.3)$ & & $45(10.0)$ & \\
\hline RespSuprt & 1,445 & $200(13.8)$ & 465 & $85(18.3)$ & $0.019^{c}$ \\
\hline OnPressors & 1,445 & $91(6.3)$ & 465 & $27(5.8)$ & $0.70^{\mathrm{c}}$ \\
\hline HemodInstab & 1,445 & $94(6.5)$ & 465 & $29(6.2)$ & $0.84^{\circ}$ \\
\hline COPD_emphysema & 1,161 & $74(6.4)$ & 440 & $54(12.3)$ & $<0.001^{e}$ \\
\hline Asthma & 1,168 & $199(17.0)$ & 442 & $100(22.6)$ & $0.010^{r}$ \\
\hline Diabetes & 1,179 & $278(23.6)$ & 450 & $164(36.4)$ & $<0.001^{c}$ \\
\hline Hypertension & 1,198 & $571(47.7)$ & 453 & $292(64.5)$ & $<0.001^{c}$ \\
\hline Coronary_artery_disease & 1,162 & $107(9.2)$ & 445 & $98(22.0)$ & $<0.001^{c}$ \\
\hline Heart_Failure & 1,162 & $105(9.0)$ & 444 & $70(15.8)$ & $<0.001^{c}$ \\
\hline Cancer & 1,202 & $164(13.6)$ & 447 & $75(16.8)$ & $0.11^{e}$ \\
\hline On_immunosuppressive_treatment & 1,209 & $119(9.8)$ & 446 & $55(12.3)$ & $0.14^{c}$ \\
\hline History_of_transplant & 1,159 & $9(0.78)$ & 443 & $10(2.3)$ & $0.014^{c}$ \\
\hline
\end{tabular}

Statistics presented as Mean $\pm \mathrm{SD}, \mathrm{N}$ (column \%).

p-values: $\mathrm{a}=\mathrm{t}$-test, $\mathrm{c}=$ Pearson's chi-square test, $\mathrm{d}=$ Fisher's Exact test. 
217 The 248 propensity-matched patients either treated with aspirin or not demonstrated no significant

218 group differences in demographics or clinical covariates. Aspirin therapy did not alter mortality (13.3\%

219 vs $15.3 \%, \mathrm{p}=0.53)$. The 444 propensity-matched patients either exposed or not to NSAIDs demonstrated

220 no significant group differences in demographics or clinical covariates. NSAID therapy did not alter

221 mortality $(7.0 \%$ vs $7.2 \%, p=0.90)$. In propensity-matched patients treated with aspirin, the incidence of

$222 \mathrm{MI}(2.0 \%$ vs $0.81 \%, \mathrm{p}=0.27)$ and VTE $(4.0 \%$ vs $1.6 \%, \mathrm{p}=0.12)$ were not significantly different, but aspirin

223 therapy was associated with an increased risk of thrombotic stroke ( $3.6 \%$ vs $0.40 \%, p=0.036)$. In

224 propensity-matched patients treated with NSAIDs, the incidence of $\mathrm{MI}(0.68 \%$ vs $0.23 \%, p=0.34), \mathrm{VTE}$

$225(2.0 \%$ vs $0.90 \%, p=0.17)$, and thrombotic stroke ( $1.1 \%$ vs $0.45 \%, p=0.27)$ was not affected. Using the

226 composite thrombotic endpoint of $\mathrm{MI}, \mathrm{VTE}$, and thrombotic stroke, both aspirin $(9.3 \%$ aspirin vs $2.8 \%$ no

227 aspirin, $p=0.005)$ and NSAID therapy ( $3.8 \%$ NSAIDs vs $1.6 \%$ no NSAIDs, $p=0.046)$ were associated with

228 signals for thrombosis (Supplemental Figure 1). Overall, there was no change in mortality in COVID-19

229 for patient treated with either aspirin (OR $0.52,95 \% \mathrm{Cl}: 0.51-1.41 ; \mathrm{p}=0.52$ ) or NSAIDs (OR $0.97,95 \% \mathrm{Cl}$ :

$230 \quad 0.58-1.62 ; p=0.90$ ) (Figure 4).

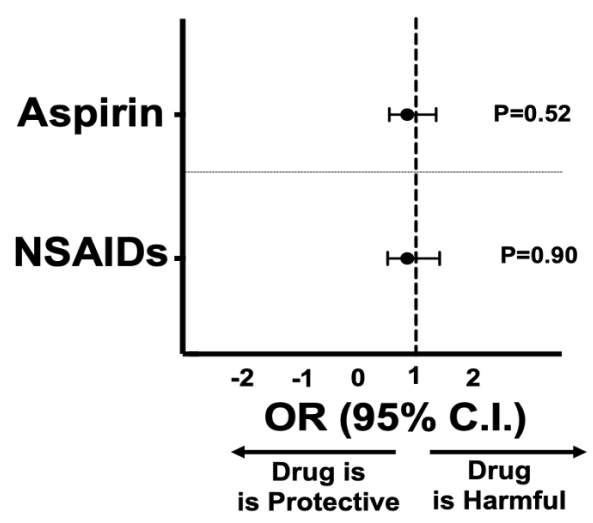

Figure 4. Mortality for Propensity-matched patients: Propensity-matched data for patients testing positive for COVD-19 and outcomes taking either $81 \mathrm{mg}$ aspirin ( $\mathrm{n}=248$ in each group) or NSAIDs ( $\mathrm{n}=444$ in each group) at the time of diagnosis. Forest plot representation of data as Odds Ratio (OR) with $95 \%$ confidence interval (C.I.) for the primary endpoint of death

232 However, both aspirin and NSAID use in COVID-19 show signals for harm with increased thrombotic risk 
233 with aspirin (OR 3.52, 95\% Cl: 1.48-8.40; $\mathrm{p}=0.005$ ) and NSAIDs (OR 2.49, 95\% Cl: 0.58-1.62; $\mathrm{p}=0.046$ ) for

234 the composite endpoint of MI, thrombotic stroke, and VTE (Supplemental Figure 2).

236 Discussion

237 In this study, we make the observation that both ACE2 and TMPRSS2 proteins which bind and ligate

238 SARS-CoV-2 are expressed in healthy human platelets. The expression of these receptors in platelets

239 does not vary significantly with age and, while numerically higher, are not strikingly different in patients

240 with CAD compared to healthy controls. The presence of known SARS-CoV-2 receptors on platelets

241 suggests the possibility that SARS-CoV-2 may directly activate platelets and contribute to thrombosis or

242 promote thrombosis indirectly by mediators secreted from platelets .

244 A recent investigation revealed platelet reactivity is enhanced in COVID-19 patients ${ }^{20,32-34}$ and appears to

245 be suppressed by the presence of high dose aspirin in vitro ${ }^{33}$. In the absence of randomized controlled

246 data for aspirin in patients with COVID-19, we conducted a propensity-matched analysis of patients

247 showing aspirin has no mortality benefit in patients with COVID-19, and, in fact, displays a slightly

248 increased signal for harm driven mostly by thrombotic stroke. Platelet reactivity data in vitro is often

249 extrapolated to suggest a risk for harm, but it is important to acknowledge that the behavior of anti-

250 platelet medications in vivo can be markedly different from in vitro studies. Our goal was to clarify this

251 concern by using real-life data with both mortality and thrombotic end points.

253 The failure to show a protective effect of the antiplatelet medication aspirin in patients with COVID-19

254 may be related to the dose administered, an insensitivity to aspirin's mechanism of platelet inhibition in

255 COVID-19, or an altered platelet phenotype as was clearly demonstrated by Manne et al. comparing

256 healthy platelets to platelets from patients with COVID-1933. Cameron et al. previously demonstrated a 
257 divergent platelet phenotype in patients with chronic arterial disease and diabetes with resistance to

258 aspirin and clopidogrel therapy in diseased but not healthy platelets ${ }^{21}$. Similarly, Liang et al

259 demonstrated in platelets from patients with diabetes, surface $\mathrm{P} 2 \mathrm{Y}_{12}$ receptors are arranged in a

260 different conformation and are impressively resistant to inhibition by clopidogrel ${ }^{35}$.

262 Elbadawi et al. reported the absolute neutrophil count and not D-dimer, a traditional biomarker

263 associated with thrombosis, is an independent predictor of thrombotic events in patients with COVID-

$26419^{36}$. The mortality benefit of dexamethasone, an immunosuppressant and anti-inflammatory

265 medication, in hospitalized patients with COVID-1937 and recent reports of immunothrombosis ${ }^{17,38-41}$ and

266 microvascular occlusion ${ }^{18,42-44}$ by multiple independent groups suggest platelets may be indirect

267 mediators of thrombosis and perhaps not the best direct targets for pharmacological intervention.

268 Contemporaneous with submission of this manuscript, a smaller, non-propensity matched study has

269 shown aspirin treatment decreased mortality that was driven by reduced ICU level care and mechanical

270 ventilatory needs but not thrombosis in patients with COVID-19. This report suggests a protective effect

271 of aspirin that is distinct from altering end-organ thrombosis ${ }^{45}$, and possibly from immune-mediated

272 acute respiratory distress syndrome (ARDS) as previously demonstrated ${ }^{46,47}$. By evaluating another anti-

273 inflammatory mechanism using patients treated with NSAIDs in parallel with aspirin in the same hospital

274 and locations in the U.S., we similarly show no effect on mortality, with all statistical models accounting

275 for any contribution of prophylactic and therapeutic heparin use in hospitalized patients and subsequent

276 outcomes.

277

278 The signal for increased composite thrombotic events in COVID-19 patients treated with aspirin was

279 surprising and driven mostly by stroke. Recent observational studies show mixed results for COVID-19-

280 related stroke risk with one small study suggesting an increased risk in younger patients ${ }^{48}$, one large 
281 study showing an overall low risk ${ }^{49}$, and one very large study paradoxically showing that COVID-19

282 infection is associated with a decreased risk of thrombotic cerebrovascular stroke $\mathrm{e}^{50}$. A mechanistic

283 explanation for our finding may be related to the known neuroprotective effect of interleukin-6 (IL-6) ${ }^{51}$

284 which is greatly elevated in systemic SARS-CoV-2 infection ${ }^{52}$ and reported to be reduced by aspirin ${ }^{53}$.

286 We show quite clearly in our study with investigators working independently of each other in different

287 regions of the U.S. that human platelets contain the SARS-CoV-2 receptors ACE2 and TMPRSS2. The

288 inter-individual expression difference of platelet ACE2 and TMPRSS2 was striking. Our overall

289 observation is consistent with the findings of Zaid et al. who identified SARS-CoV-2 mRNA in human

290 platelets implying a mechanism of entry must exist, and then a report by Zhang et al. who identified

291 ACE2 on human platelets ${ }^{20,54}$. Our data are at odds with Manne et al. who failed to detect ACE2 protein

292 in platelets by immunoblotting using white blood cells (WBC) as a positive control ${ }^{33}$. Notably, Manne et

293 al. employed a CD45 depletion step on isolated platelets to eliminate the possibility of WBC

294 contamination prior to immunoblotting. CD45 is also present on platelets, and we previously

295 demonstrated this step decreases the platelet yield available for immunoblotting ${ }^{22}$. Lastly, Nassa et al.

296 have very elegantly shown that the platelet transcriptome and proteome are dynamic and often mRNA

297 to protein concordance is not observed but, rather, dependent on external platelet cues ${ }^{55}$. Overall, our

298 data are congruent with Koupenova et al. suggesting that the ssRNA virus SARS-CoV-2 may behave

299 similarly to the ssRNA influenza virus by utilizing platelets to modulate immune function that ultimately

300 may lead to immunothrombosis ${ }^{16}$. 


\section{Study Limitations}

306 The observational nature of this study from just two hospitals has intrinsic limitations, and the small

307 patient sample to allow for propensity matching limits generalizability of our findings. A few patients

308 testing positive for SARS-CoV-2 were ambulatory and we relied on physician prescriptions making it

309 impossible to confirm compliance to aspirin therapy.

\section{Conclusions}

312 SARS-CoV-2 high affinity receptors are present in platelets from healthy individuals. This finding crucially

313 suggests platelets may be involved in COVID-19 pathogenesis and the observed thrombotic phenotype.

314 However, our real-world clinical data suggests regular intake of low dose aspirin does not protect

315 against adverse thrombotic events or death in COVID-19 patients. Platelets are fastidious components

316 of the circulatory system with a wide range of critical functions, including contributing to

317 immunoinflammatory host responses. Thus, targeting platelet thrombotic function may alter its roles in

318 other domains. The nuanced mechanisms of thrombosis in COVID-19 may be unique and deserves

319 further investigation. The use of traditional antiplatelet agents may not protect against thrombotic

320 events or mortality in COVID-19, but, in fact, cause harm. The awareness of this potential harm and role

321 of randomized controlled drug trials in assessing the suitability of antiplatelet agents in COVID-19 is

322 critical. 
330 Study design (AS, SJC, RB, AE, AK, JF, LGS, MKC, HK, JPI, MK, SK, TM, KRM)

331 Data collection (AS, SJC, RB, MKC, HK, JPI, MG, MK, JEF, JRB, EH, AA)

332 Data analysis (AS, SJC, AE, MKC, HK, JPI, MK, JEF, JRB, EH, SK)

333 Data interpretation (AE, AK, JF, LGS, MKC, HK, JPI, MG, MK, JEF, JRB, EH, AA, SK)

334 Figures (AS, SJC, MG, MK, JEF, JRB, EH, AA, SK)

335 Literature (AS, SJC, RB, AE, AK, JF, LGS, MKC, HK, MK, JEF, JRB, EH, SK, TM KRM)

336 Writing (AS, SJC, RB, AE AK, JF, MKC, HK, JPI, MG, MK, JEF, SK, TM, KRM)

337 All authors gave final approval for this version of the manuscript to be published.

\section{Acknowledgements}

340 Financial support from NHLBI K08HL128856 and NHLBI LRPHL120200 to SJC and R01 HL143402 to KRM,

341 R01 HL153235 and CTSA grant (UMCCTS funded by NCATS: grant UL1TR001453, TL1TR00454, and

342 KL2TR001455) to MK, and AHA COVID19 Rapid Response Award to JEF.

\section{Declaration of Interests}

345 None of the authors have any relevant conflicting financial, personal, or professional relationships. 


\section{References}

$3601 \quad$ Klok, F. A. et al. Incidence of thrombotic complications in critically ill ICU patients with COVID-19. Thromb Res 191, 145-147, doi:10.1016/j.thromres.2020.04.013 (2020). Merkler, A. E. et al. Risk of Ischemic Stroke in Patients With Coronavirus Disease 2019 (COVID19) vs Patients With Influenza. JAMA Neurol, doi:10.1001/jamaneurol.2020.2730 (2020).

3 Helms, J. et al. High risk of thrombosis in patients with severe SARS-CoV-2 infection: a multicenter prospective cohort study. Intensive Care Med 46, 1089-1098, doi:10.1007/s00134020-06062-x (2020).

4 Shang, J. et al. Cell entry mechanisms of SARS-CoV-2. Proc Natl Acad Sci U S A 117, 11727-11734, doi:10.1073/pnas.2003138117 (2020).

5 Hoffmann, M. et al. SARS-CoV-2 Cell Entry Depends on ACE2 and TMPRSS2 and Is Blocked by a Clinically Proven Protease Inhibitor. Cell 181, 271-280.e278, doi:10.1016/j.cell.2020.02.052 (2020).

6 Saheb Sharif-Askari, N. et al. Airways Expression of SARS-CoV-2 Receptor, ACE2, and TMPRSS2 Is Lower in Children Than Adults and Increases with Smoking and COPD. Mol Ther Methods Clin Dev 18, 1-6, doi:10.1016/j.omtm.2020.05.013 (2020).

7 Sungnak, W. et al. SARS-CoV-2 entry factors are highly expressed in nasal epithelial cells together with innate immune genes. Nat Med 26, 681-687, doi:10.1038/s41591-020-0868-6 (2020).

8 Qi, F., Qian, S., Zhang, S. \& Zhang, Z. Single cell RNA sequencing of 13 human tissues identify cell types and receptors of human coronaviruses. Biochem Biophys Res Commun 526, 135-140, doi:10.1016/j.bbrc.2020.03.044 (2020).

9 Zhao, Y. et al. Single-Cell RNA Expression Profiling of ACE2, the Receptor of SARS-CoV-2. Am J Respir Crit Care Med 202, 756-759, doi:10.1164/rccm.202001-0179LE (2020).

10 Hamming, l. et al. Tissue distribution of ACE2 protein, the functional receptor for SARS coronavirus. A first step in understanding SARS pathogenesis. J Pathol 203, 631-637, doi:10.1002/path.1570 (2004).

11 Bertram, S. et al. Influenza and SARS-coronavirus activating proteases TMPRSS2 and HAT are expressed at multiple sites in human respiratory and gastrointestinal tracts. PLoS One 7, e35876, doi:10.1371/journal.pone.0035876 (2012).

405 He, L. et al. Pericyte-specific vascular expression of SARS-CoV-2 receptor ACE2 - implications for microvascular inflammation and hypercoagulopathy in COVID-19 patients. bioRxiv, 2020.2005.2011.088500, doi:10.1101/2020.05.11.088500 (2020).

13 Harmer, D., Gilbert, M., Borman, R. \& Clark, K. L. Quantitative mRNA expression profiling of ACE 2, a novel homologue of angiotensin converting enzyme. FEBS Lett 532, 107-110, doi:10.1016/s0014-5793(02)03640-2 (2002).

14 Hikmet, F. et al. The protein expression profile of ACE2 in human tissues. Mol Syst Biol 16, e9610-e9610, doi:10.15252/msb.20209610 (2020).

15 Liang, W. et al. Diarrhoea may be underestimated: a missing link in 2019 novel coronavirus. Gut 69, 1141-1143, doi:10.1136/gutjnl-2020-320832 (2020).

16 Koupenova, M. et al. The role of platelets in mediating a response to human influenza infection. Nat Commun 10, 1780, doi:10.1038/s41467-019-09607-x (2019).

17 Zuo, Y. et al. Neutrophil extracellular traps in COVID-19. JCI Insight 5, doi:10.1172/jci.insight.138999 (2020).

18 Goshua, G. et al. Endotheliopathy in COVID-19-associated coagulopathy: evidence from a singlecentre, cross-sectional study. Lancet Haematol 7, e575-e582, doi:10.1016/S2352-

3026(20)30216-7 (2020). 
$406 \quad 19$

407

$408 \quad 20$

409

410

411

412

413

414

415

416

417

418

419

420

421

422

423

424

425

426

427

428

429

430

431

432

433

434

435

436

437

438

439

440

441

442

443

444

445

446

447

448

449

450

451

452

453

Manne, B. K. et al. Platelet Gene Expression and Function in COVID-19 Patients. Blood, doi:10.1182/blood.2020007214 (2020).

20 Zaid, Y. et al. Platelets Can Associate with SARS-Cov-2 RNA and Are Hyperactivated in COVID-19. Circ Res, doi:10.1161/CIRCRESAHA.120.317703 (2020).

21 Cameron, S. J. et al. Platelet Extracellular Regulated Protein Kinase 5 Is a Redox Switch and Triggers Maladaptive Platelet Responses and Myocardial Infarct Expansion. Circulation 132, 4758, doi:10.1161/CIRCULATIONAHA.115.015656 (2015).

22 Schmidt, R. A. et al. The platelet phenotype in patients with ST-segment elevation myocardial infarction is different from non-ST-segment elevation myocardial infarction. Transl Res 195, 112, doi:10.1016/j.trsl.2017.11.006 (2018).

23 Lemme, M. et al. Atrial-like Engineered Heart Tissue: An In Vitro Model of the Human Atrium. Stem Cell Reports 11, 1378-1390, doi:10.1016/j.stemcr.2018.10.008 (2018).

24 Li, F., Thomas, L. E. \& Li, F. Addressing Extreme Propensity Scores via the Overlap Weights. Am J Epidemiol 188, 250-257, doi:10.1093/aje/kwy201 (2019).

25 Ding, P. \& VanderWeele, T. J. Sensitivity Analysis Without Assumptions. Epidemiology 27, 368377, doi:10.1097/EDE.0000000000000457 (2016).

26 Towler, P. et al. ACE2 X-ray structures reveal a large hinge-bending motion important for inhibitor binding and catalysis. J Biol Chem 279, 17996-18007, doi:10.1074/jbc.M311191200 (2004).

27 Chen, R. et al. Glycoproteomics analysis of human liver tissue by combination of multiple enzyme digestion and hydrazide chemistry. J Proteome Res 8, 651-661, doi:10.1021/pr8008012 (2009).

28 Glasgow, A. et al. Engineered ACE2 receptor traps potently neutralize SARS-CoV-2. Proc Natl Acad Sci U S A, doi:10.1073/pnas.2016093117 (2020).

29 Alenina, N. \& Bader, M. ACE2 in Brain Physiology and Pathophysiology: Evidence from Transgenic Animal Models. Neurochem Res 44, 1323-1329, doi:10.1007/s11064-018-2679-4 (2019).

30 Li, M., Chen, L., Zhang, J., Xiong, C. \& Li, X. The SARS-CoV-2 receptor ACE2 expression of maternal-fetal interface and fetal organs by single-cell transcriptome study. PLoS One 15, e0230295, doi:10.1371/journal.pone.0230295 (2020).

31 Monteil, V. et al. Inhibition of SARS-CoV-2 Infections in Engineered Human Tissues Using ClinicalGrade Soluble Human ACE2. Cell 181, 905-913 e907, doi:10.1016/j.cell.2020.04.004 (2020).

32 Hottz, E. D. et al. Platelet activation and platelet-monocyte aggregate formation trigger tissue factor expression in patients with severe COVID-19. Blood 136, 1330-1341, doi:10.1182/blood.2020007252 (2020).

33 Manne, B. K. et al. Platelet gene expression and function in patients with COVID-19. Blood 136, 1317-1329, doi:10.1182/blood.2020007214 (2020).

34 Barrett, T. J. et al. Platelet and Vascular Biomarkers Associate With Thrombosis and Death in Coronavirus Disease. Circ Res 127, 945-947, doi:10.1161/CIRCRESAHA.120.317803 (2020).

$35 \mathrm{Hu}$, L. et al. Platelets Express Activated P2Y12 Receptor in Patients With Diabetes Mellitus. Circulation 136, 817-833, doi:10.1161/CIRCULATIONAHA.116.026995 (2017).

36 Elbadawi, A. et al. Incidence and Outcomes of Thrombotic Events in Symptomatic Patients With COVID-19. Arteriosclerosis, thrombosis, and vascular biology, ATVBAHA120315304, doi:10.1161/ATVBAHA.120.315304 (2020).

37 Group, R. C. et al. Dexamethasone in Hospitalized Patients with Covid-19 - Preliminary Report. N Engl J Med, doi:10.1056/NEJMoa2021436 (2020).

38 Barnes, B. J. et al. Targeting potential drivers of COVID-19: Neutrophil extracellular traps. J Exp Med 217, doi:10.1084/jem.20200652 (2020). 
Wang, J. et al. Excessive Neutrophils and Neutrophil Extracellular Traps in COVID-19. Front Immunol 11, 2063, doi:10.3389/fimmu.2020.02063 (2020). Veras, F. P. et al. SARS-CoV-2-triggered neutrophil extracellular traps mediate COVID-19 pathology. J Exp Med 217, doi:10.1084/jem.20201129 (2020). Skendros, P. et al. Complement and tissue factor-enriched neutrophil extracellular traps are key drivers in COVID-19 immunothrombosis. J Clin Invest 130, 6151-6157, doi:10.1172/JCI141374 (2020). Respiratory Failure and Coagulopathy. Circulation 142, 1176-1189, doi:10.1161/CIRCULATIONAHA.120.048488 (2020). von der Thusen, J. H. et al. Case report: a fatal combination of hemophagocytic lymphohistiocytosis with extensive pulmonary microvascular damage in COVID-19 pneumonia. J Hematop, 1-5, doi:10.1007/s12308-020-00423-7 (2020). doi:10.1007/s10456-020-09753-7 (2020).

Chow, J. H. et al. Aspirin Use is Associated with Decreased Mechanical Ventilation, ICU Admission, and In-Hospital Mortality in Hospitalized Patients with COVID-19. Anesth Analg,

46 Erlich, J. M., Talmor, D. S., Cartin-Ceba, R., Gajic, O. \& Kor, D. J. Prehospitalization antiplatelet therapy is associated with a reduced incidence of acute lung injury: a population-based cohort study. Chest 139, 289-295, doi:10.1378/chest.10-0891 (2011).

47 Kor, D. J. et al. Effect of Aspirin on Development of ARDS in At-Risk Patients Presenting to the Emergency Department: The LIPS-A Randomized Clinical Trial. JAMA 315, 2406-2414, doi:10.1001/jama.2016.6330 (2016).

48 Ellul, M. A. et al. Neurological associations of COVID-19. Lancet Neurol 19, 767-783, doi:10.1016/S1474-4422(20)30221-0 (2020).

49 Shahjouei, S. et al. Risk of stroke in hospitalized SARS-CoV-2 infected patients: A multinational study. EBioMedicine 59, 102939, doi:10.1016/j.ebiom.2020.102939 (2020).

50 Bekelis, K. et al. Ischemic Stroke Occurs Less Frequently in Patients With COVID-19: A Multicenter Cross-Sectional Study. Stroke, STROKEAHA120031217, doi:10.1161/STROKEAHA.120.031217 (2020).

51 Smith, C. J. et al. Peak plasma interleukin-6 and other peripheral markers of inflammation in the first week of ischaemic stroke correlate with brain infarct volume, stroke severity and long-term outcome. BMC Neurol 4, 2, doi:10.1186/1471-2377-4-2 (2004).

52 Leisman, D. E. et al. Cytokine elevation in severe and critical COVID-19: a rapid systematic review, meta-analysis, and comparison with other inflammatory syndromes. Lancet Respir Med, doi:10.1016/S2213-2600(20)30404-5 (2020).

53 Haynes, D. R., Wright, P. F., Gadd, S. J., Whitehouse, M. W. \& Vernon-Roberts, B. Is aspirin a prodrug for antioxidant and cytokine-modulating oxymetabolites? Agents Actions 39, 49-58, doi:10.1007/BF01975714 (1993).

54 Zhang, S. et al. SARS-CoV-2 binds platelet ACE2 to enhance thrombosis in COVID-19. J Hematol Oncol 13, 120, doi:10.1186/s13045-020-00954-7 (2020). results in functionally relevant proteome modifications. Sci Rep 8, 498, doi:10.1038/s41598-01718985-5 (2018). 
Figure 1. Expression of ACE2 and TMPRSS2 in Platelets by Confocal Microscopy. Platelets isolated from venous blood of healthy individuals was stained for $1 \mathrm{~h}$ with the following antibodies: CD41 (platelet-specific marker), ACE2, TMPRSS2, and DAPI to eliminate any DNA components. Mounted slides were resolved by confocal fluorescent microscopy using a 100x objective lens. Images are representative of $n=6$ donors for $A C E 2$ and $n=3$ for TMPRSS2. Each image represents a different donor. The scale bar is noted.

Figure 2. A. Expression of TMPRSS2 in Platelets: Washed platelets from healthy individuals (mean age $40.1 \pm 2.8$ years, $n=20$ ) were isolated and proteins separate by SDS-PAGE with molecular weight shown in KiloDaltons (KDa). Immunoblotting was conducted an using an anti-TMPRSS2 antibody or anti-tubulin immunoblotting as a loading control. The ratio of protein to loading control is expressed as a function of age and the correlation coefficient is noted $(r \pm 95 \% \mathrm{Cl}, \mathrm{P}=0.30)$. Human brain lysate served as a positive control for TMPRSS2 migrating at the expected molecular weight ( $50 \mathrm{KDa})$. Data shown are representative of 20 healthy individuals ( 10 male and 10 female) and 10 patients with coronary artery disease (CAD). The mean ratio of TMPRSS2/Tubulin \pm SEM is noted, $P=0.145$ between healthy and CAD by Mann Whitney $U$ ). B. Expression of ACE2 in Platelets: Washed platelets from healthy individuals (mean age $40.1 \pm 2.8$ years, $n=20$ ) were isolated and proteins separate by SDS-PAGE with molecular weight shown in KiloDaltons (KDa). Lane 1 is human platelet lysate, lane 2 is human brain lysate, lane 3 is human placenta lysate, lane 4 is lysate from engineered human heart tissue. Immunoblotting was conducted using an using anti-ACE2 antibody. Anti-tubulin and anti-GAPDH are loading controls. ACE2 migrates at the expected molecular weight ( $100 \mathrm{KDa}$ ) shown by an arrowhead with glycosylated forms indicated by *. The ratio of ACE2 protein to loading control is expressed as a function of age and the correlation coefficient is noted $(r \pm 95 \% \mathrm{Cl}, \mathrm{P}=0.79)$. Data shown are representative of 20 healthy individuals ( 10 male and 10 female) and 10 patients with coronary artery disease (CAD). The mean ratio of ACE2/Tubulin \pm SEM is noted, $P=0.112$ between healthy and CAD by Mann Whitney $U$ ).

Figure 3. Patients Testing Positive for SARS-CoV-2 taking Aspirin or NSAIDs. Patients testing positive for a SARS-CoV-2 amplicon at two Cleveland Clinic hospitals were evaluated. Patients initiated with aspirin or NSAID therapy or continuing aspirin or NSAID if admitted to the hospital were included in this study. Clinical variables in each group where then re-evaluated following careful propensity matching .

Table 1. Characteristics of Population taking Aspirin Therapy: Unadjusted data are for patients testing positive for SARS-CoV-2 not taking aspirin or with established aspirin therapy or initiated with low dose aspirin at the time of diagnosis.

Table 2. Characteristics of Population taking NSAID Therapy: Unadjusted data are for patients testing positive for SARS-CoV-2 not taking NSAID or with established NSAID therapy or initiated with NSAID at the time of diagnosis.

Figure 4. Mortality for Propensity-matched patients: Propensity-matched data for patients testing positive for COVD-19 and outcomes taking either $81 \mathrm{mg}$ aspirin ( $\mathrm{n}=248$ in each group) or NSAIDs ( $\mathrm{n}=444$ in each group) at the time of diagnosis. Forest plot representation of data as Odds Ratio (OR) with $95 \%$ confidence interval (C.I.) for the primary endpoint of death. 


\section{Figures}
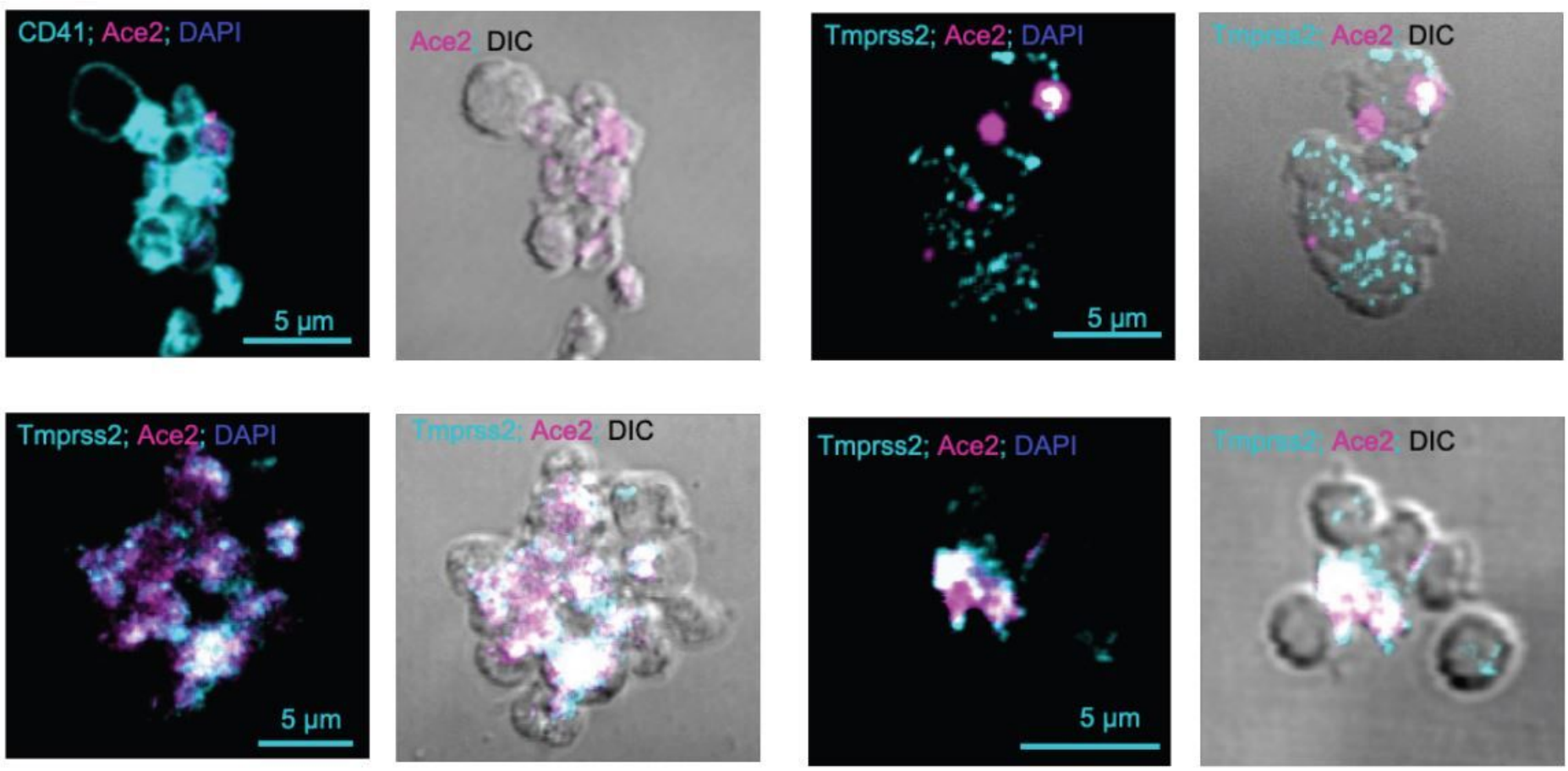

\section{Figure 1}

Expression of ACE2 and TMPRSS2 in Platelets by Confocal Microscopy. Platelets isolated from venous blood of healthy individuals was stained for $1 \mathrm{~h}$ with the following antibodies: CD41 (platelet-specific marker), ACE2, TMPRSS2, and DAPI to eliminate any DNA components. Mounted slides were resolved by confocal fluorescent microscopy using a $100 x$ objective lens. Images are representative of $n=6$ donors for ACE2 and $n=3$ for TMPRSS2. Each image represents a different donor. The scale bar is noted. 

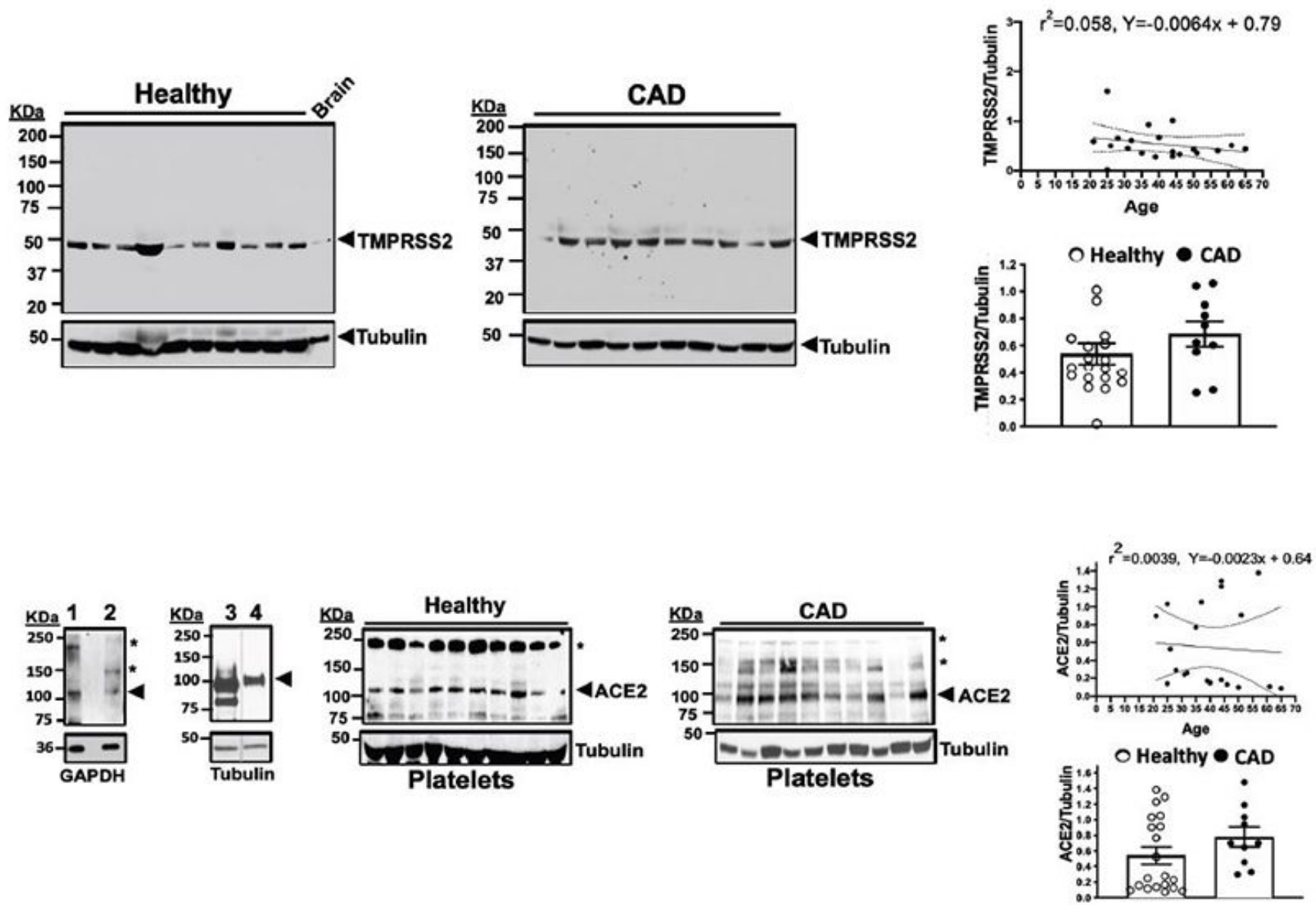

\section{Figure 2}

A. Expression of TMPRSS2 in Platelets: Washed platelets from healthy individuals (mean age $40.1 \pm 2.8$ years, $n=20$ ) were isolated and proteins separate by SDS-PAGE with molecular weight shown in KiloDaltons (KDa). Immunoblotting was conducted an using an anti-TMPRSS2 antibody or anti-tubulin immunoblotting as a loading control. The ratio of protein to loading control is expressed as a function of age and the correlation coefficient is noted $(r \pm 95 \% \mathrm{Cl}, \mathrm{P}=0.30)$. Human brain lysate served as a positive control for TMPRSS2 migrating at the expected molecular weight ( 50 KDa). Data shown are representative of 20 healthy individuals ( 10 male and 10 female) and 10 patients with coronary artery disease (CAD). The mean ratio of TMPRSS2/Tubulin \pm SEM is noted, $P=0.145$ between healthy and CAD by Mann Whitney U). B. Expression of ACE2 in Platelets: Washed platelets from healthy individuals (mean age $40.1 \pm 2.8$ years, $n=20$ ) were isolated and proteins separate by SDS-PAGE with molecular weight shown in KiloDaltons (KDa). Lane 1 is human platelet lysate, lane 2 is human brain lysate, lane 3 is human placenta lysate, lane 4 is lysate from engineered human heart tissue. Immunoblotting was conducted using an using anti-ACE2 antibody. Anti-tubulin and anti-GAPDH are loading controls. ACE2 migrates at the expected molecular weight ( $100 \mathrm{KDa}$ ) shown by an arrowhead with glycosylated forms indicated by *. The ratio of ACE2 protein to loading control is expressed as a function of age and the 
correlation coefficient is noted $(r \pm 95 \% \mathrm{Cl}, \mathrm{P}=0.79)$. Data shown are representative of 20 healthy individuals (10 male and 10 female) and 10 patients with coronary artery disease (CAD). The mean ratio of ACE2/Tubulin \pm SEM is noted, $P=0.112$ between healthy and CAD by Mann Whitney $U$ ).

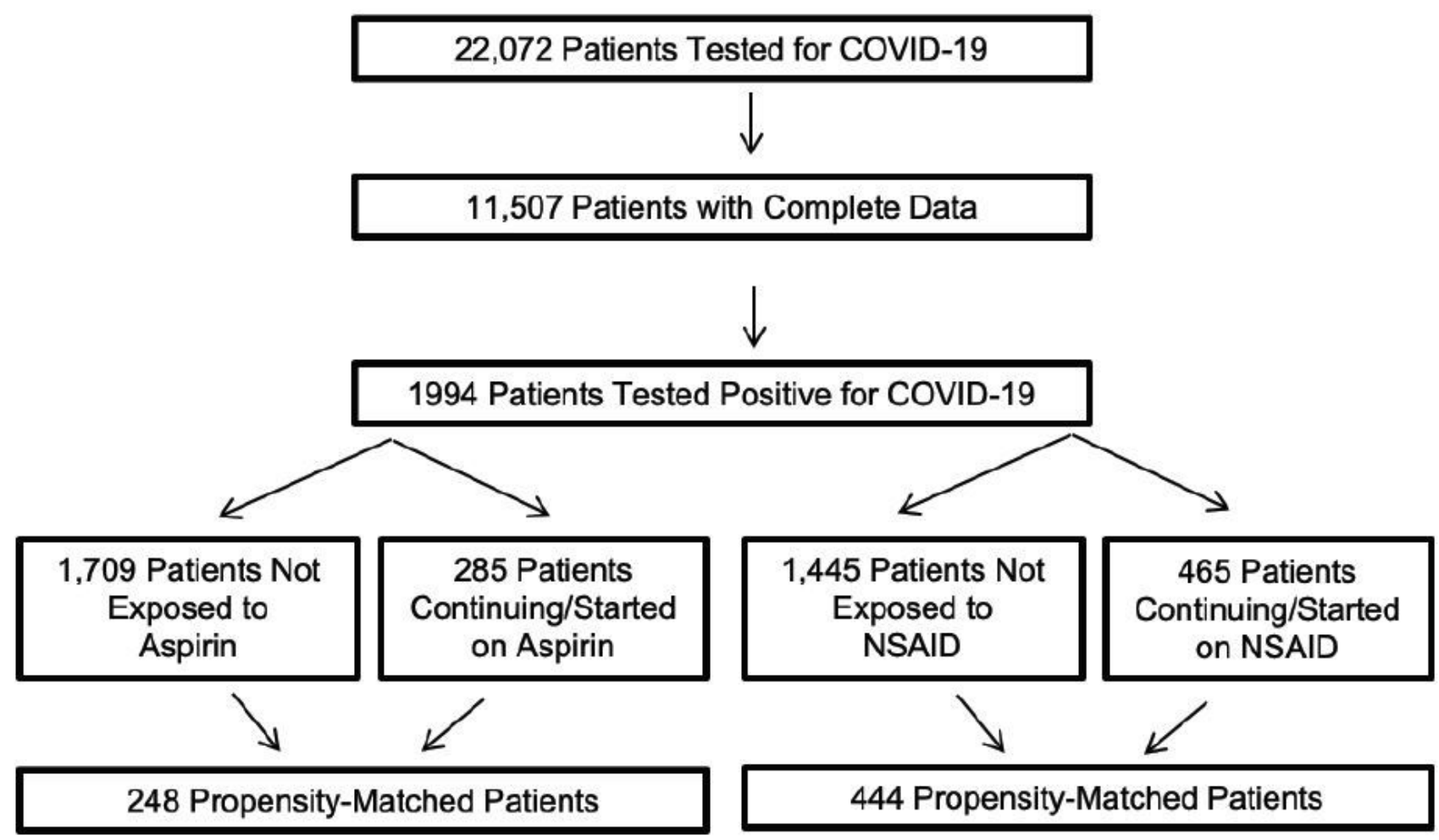

Figure 3

Patients Testing Positive for SARS-CoV-2 taking Aspirin or NSAIDs. Patients testing positive for a SARSCoV-2 amplicon at two Cleveland Clinic hospitals were evaluated. Patients initiated with aspirin or NSAID therapy or continuing aspirin or NSAID if admitted to the hospital were included in this study. Clinical variables in each group where then re-evaluated following careful propensity matching . 


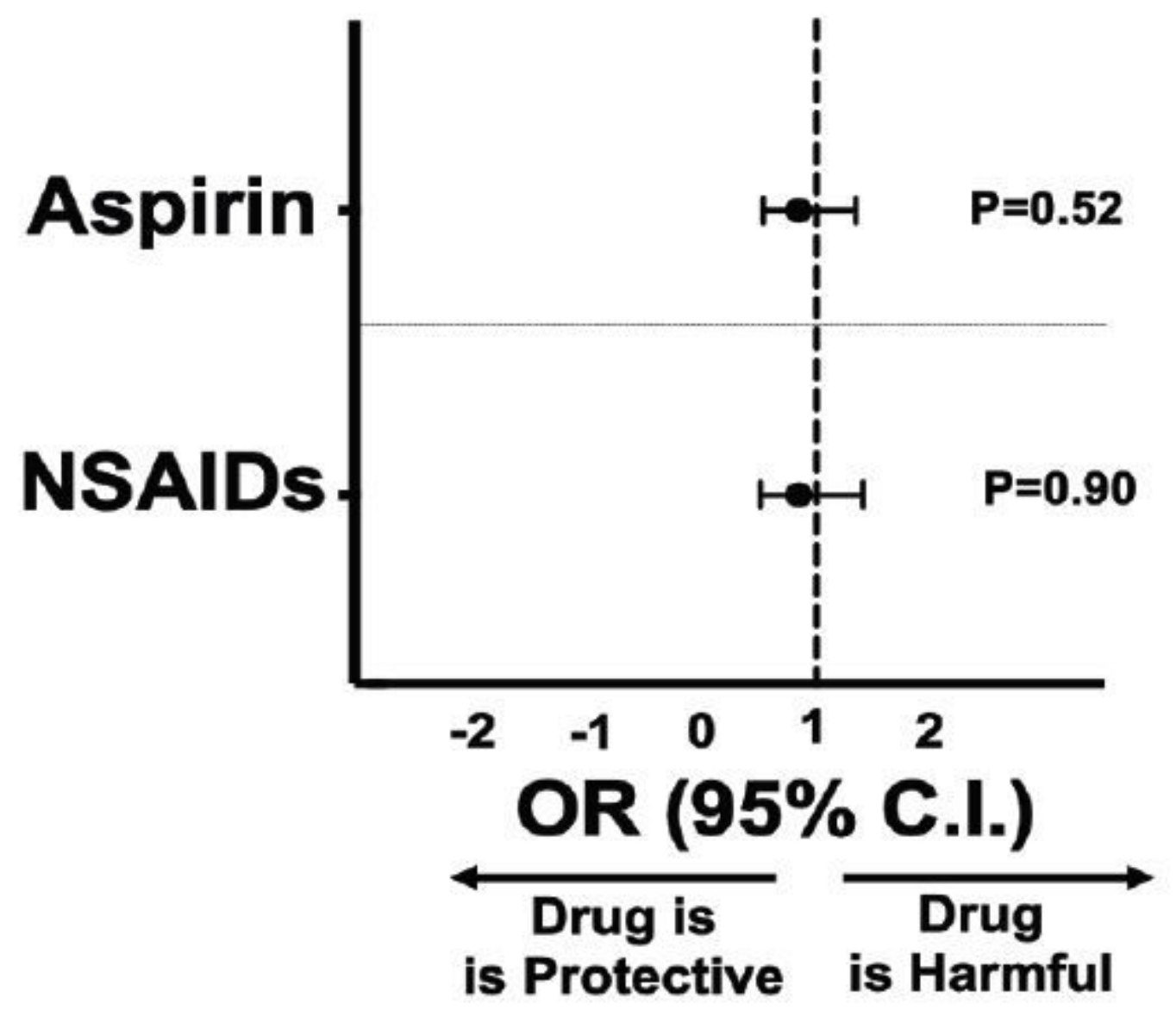

Figure 4

Mortality for Propensity-matched patients: Propensity-matched data for patients testing positive for COVD-19 and outcomes taking either $81 \mathrm{mg}$ aspirin ( $\mathrm{n}=248$ in each group) or NSAIDs ( $\mathrm{n}=444$ in each group) at the time of diagnosis. Forest plot representation of data as Odds Ratio (OR) with $95 \%$ confidence interval (C.I.) for the primary endpoint of death.

\section{Supplementary Files}

This is a list of supplementary files associated with this preprint. Click to download.

- SupplementalFile1221.20.pdf 